EPJ manuscript No.

(will be inserted by the editor)

\title{
Re-evaluation of neutrino mixing pattern according to latest $\mathrm{T} 2 \mathrm{~K}$ result
}

\author{
Ya-juan Zheng ${ }^{1,2}$ and Bo-Qiang Ma ${ }^{1,3 a}$ \\ 1 School of Physics and State Key Laboratory of Nuclear Physics and Technology, Peking University, Beijing 100871, China \\ 2 Department of Physics, Shandong University, Jinan, Shandong 250100, China \\ 3 Center for High Energy Physics, Peking University, Beijing 100871, China
}

Received: date / Revised version: date

\begin{abstract}
We re-evaluate neutrino mixing patterns according to the latest $\mathrm{T} 2 \mathrm{~K}$ result for a larger mixing angle $\theta_{13}$, and find that the PMNS mixing matrix has larger deviations from bimaximal (BM) and tribimaximal (TB) mixing patterns than previously expected. We also find that several schemes connecting PMNS and CKM mixing matrices can accommodate the latest T2K result nicely. As necessary updates to former works, we make new triminimal expansions of PMNS mixing matrix based on BM and TB mixing patterns. We also propose a new mixing pattern with a self-complementary relation between the mixing angles $\theta_{12}^{\nu}+\theta_{13}^{\nu} \simeq 45^{\circ}$, and find such a new mixing pattern in leading order can provide a rather good description of the data.
\end{abstract}

PACS. $14.60 . \mathrm{Pq}-12.15 . \mathrm{Ff}-14.60 . \mathrm{Lm}$

\section{Introduction}

Neutrino oscillations observed in the solar [1], atmospheric [2], reactor [3] and accelerator [4] neutrino experiments have provided plentiful information on neutrino masses and neutrino mixings. Among the knowns and unknowns of neutrino physics, the nonzero but nontrivial smallest mixing angle $\theta_{13}$ has received a lot of attentions, therefore the measurement of $\theta_{13}$ is important to enrich our understanding of neutrino properties. It may lead to the measurement of $\mathrm{CP}$ violation in the lepton sector and the study of its origin. In addition, the precision measurement of $\theta_{13}$ would greatly enhance our knowledge to the solar, atmospheric, the secondary effects of long baseline, and the supernova neutrino oscillations as well.

In the last decade, international collaborations have been established to focus on the measurement of $\theta_{13}$, e.g., Double Chooz in France, Reactor Experiment for Neutrino Oscillations (RENO) in South Korea, Daya Bay Experiment in China, $\mathrm{NO} \nu \mathrm{A}$ in United States, and Tokai to Kamioka (T2K) experiment in Japan. With the appearance of the latest result from $\mathrm{T} 2 \mathrm{~K}[5]$, which indicates a relatively large $\theta_{13}$, we need to re-evaluate our understanding on neutrino masses and mixings, especially on the neutrino mixing pattern $[6,7]$.

Ever since the establishment of neutrino oscillation, theorists have been working on the explanation of neutrino flavor mixings. From bimaximal (BM) mixing, trimaximal (TB) mixing to the tribimaximal mixing, various neutrino mixing patterns have been brought up and later evaluated by the experimental data. On the other hand, the underlying unification and symmetry concerning leptons and quarks are speculated from some clues of low energy experimental data, such as the quark-lepton complementarity (QLC) [8-19]. As the first result from T2K offers new bounds on the smallest neutrino mixing angles, we aim to make an analysis on the implications and consequences from the observation of a nonzero and also not so small value of $\theta_{13}$.

The remaining part of this paper is organized as follows. In Sec. 2, we check experimental status of quark and lepton mixings based on previous fits together with the latest T2K result of a larger mixing angle $\theta_{13}$. It is remarkable that the deviations of the lepton mixing matrix from bimaximal and tribimaximal mixing patterns are expressed in terms of the Wolfenstein parameter $\lambda$ of quark mixing matrix. Then we examine in Sec. 2.1 several schemes connecting the mixing matrices of quarks and leptons, and find that some of them are possible to accommodate the latest T2K data. In Sec. 3, as a necessary update, we make new expansions of PMNS mixing matrix based on both bimaximal and tribimaximal mixing patterns, with a more reasonable hierarchy structure in powers of the Wolfenstein parameter

a e-mail: mabq@pku.edu.cn 
$\lambda$ in the quark mixing matrix. In Sec. 4 from the phenomenology point of view, we propose a new mixing pattern with a self-complementary relation between the mixing angles $\theta_{12}^{\nu}+\theta_{13}^{\nu} \simeq 45^{\circ}$. The new mixing matrix is more closer to the data than previously known matrices such as BM and TB mixing patterns. Then we provide a summary in Sec. 5 .

\section{Experimental status of quark and lepton mixings}

Quark and lepton mixings are phenomenologically described by the Cabibbo-Kobayashi-Maskawa (CKM) [20,21] matrix and Pontecorvo-Maki-Nakagawa-Sakata (PMNS) [22,23] matrix respectively. The standard parameterization [24] of quark and lepton mixing matrices is expressed by three mixing angles $\theta_{12}, \theta_{13}, \theta_{23}$ and one CP-violating phase angle $\delta$. As shown below, the elements of the mixing matrix in the first row and third column adopt very simple forms

$$
V=\left(\begin{array}{ccc}
c_{12} c_{13} & s_{12} c_{13} & s_{13} e^{-i \delta} \\
-c_{12} s_{23} s_{13} e^{i \delta}-s_{12} c_{23} & -s_{12} s_{23} s_{13} e^{i \delta}+c_{12} c_{23} & s_{23} c_{13} \\
-c_{12} c_{23} s_{13} e^{i \delta}+s_{12} s_{23} & -s_{12} c_{23} s_{13} e^{i \delta}-c_{12} s_{23} & c_{23} c_{13}
\end{array}\right),
$$

where $s_{i j}=\sin \theta_{i j}, c_{i j}=\cos \theta_{i j}(i, j=1,2,3)$. For describing the quark mixing matrix $V_{\mathrm{CKM}}$, another simple form of parametrization, i.e., the Wolfenstein parametrization was proposed with the defination that $s_{12}=\lambda, s_{23}=A \lambda^{2}$ and $s_{13} e^{i \delta}=A \lambda^{3}(\rho+i \eta)[25]$. Its explicit form at the accuracy of $\mathcal{O}\left(\lambda^{4}\right)$ is

$$
V_{\mathrm{CKM}}=\left(\begin{array}{ccc}
1-\frac{1}{2} \lambda^{2} & \lambda & A \lambda^{3}(\rho-i \eta) \\
-\lambda & 1-\frac{1}{2} \lambda^{2} & A \lambda^{2} \\
A \lambda^{3}(1-\rho-i \eta) & -A \lambda^{2} & 1
\end{array}\right)+\mathcal{O}\left(\lambda^{4}\right),
$$

and we adopt the following inputs given by the Particle Data Group [26]:

$$
\lambda=0.2257_{-0.0010}^{+0.0009}, \quad A=0.814_{-0.022}^{+0.021}, \quad \bar{\rho}=0.135_{-0.016}^{+0.031}, \quad \bar{\eta}=0.349_{-0.017}^{+0.015},
$$

where $\bar{\rho}=\rho-\frac{1}{2} \rho \lambda^{2}+O\left(\lambda^{4}\right)$ and $\bar{\eta}=\eta-\frac{1}{2} \eta \lambda^{2}+O\left(\lambda^{4}\right)$. The corresponding quark mixing angles could be obtained by calculating the moduli of the mixing matrix elements $\theta_{12}^{q}=13.04_{-0.059^{\circ}}^{\circ+0.053^{\circ}}, \theta_{23}^{q}=2.37_{-0.085^{\circ}}^{\circ+0.081^{\circ}}$ and $\theta_{13}^{q}=0.20_{-0.020^{\circ}}^{\circ+0.023^{\circ}}$ [27]. One advantage of the Wolfenstein parametrization is that one may estimate the order of magnitude of any element from the hierarchical feature of $\lambda$. It is natural to recall that the deviation of $V_{\mathrm{CKM}}$ can be considered as expansions in orders of $\lambda$ from the unit matrix $I$. To assume firstly that the $V_{\text {CKM }}$ quark mixing matrix takes the form of unit matrix, it is easy to get three $0^{\circ}$ quark mixing angles in the unit mixing pattern. Hence, we can obtain the corresponding deviation of each mixing angle:

$$
\begin{cases}\Delta_{23}^{I}=\left|\theta_{23}^{q}-\theta_{23}^{I}\right| \simeq 2.37^{\circ}, & \sin \Delta_{23}^{I} \sim \mathcal{O}\left(\lambda^{2}\right), \\ \Delta_{12}^{I}=\left|\theta_{12}^{q}-\theta_{12}^{I}\right| \simeq 13.04^{\circ}, & \sin \Delta_{12}^{I} \sim \mathcal{O}(\lambda), \\ \Delta_{13}^{I}=\left|\theta_{13}^{q}-\theta_{13}^{I}\right| \simeq 0.20^{\circ}, & \sin \Delta_{13}^{I} \sim \mathcal{O}\left(\lambda^{3}\right),\end{cases}
$$

where $\lambda \simeq 0.2$ denotes the Wolfenstein parameter.

For the neutrino sector, with the latest global fit of experimental data given in $[28,29]$, the three mixing angles of the PMNS mixing matrix $U_{\mathrm{PMNS}}$ read as

$$
\begin{gathered}
\sin ^{2} \theta_{12}=0.312\left(1_{-0.109}^{+0.128}\right)(2 \sigma), \\
\sin ^{2} \theta_{23}=0.466\left(1_{-0.215}^{+0.292}\right)(2 \sigma), \\
\sin ^{2} \theta_{13}=0.016 \pm 0.010(1 \sigma),
\end{gathered}
$$

which indicate that

$$
\theta_{12}^{\nu} \simeq 33.957_{-2.143^{\circ}}^{\circ+2.434^{\circ}}, \quad \theta_{23}^{\nu} \simeq 43.050_{-5.834^{\circ}}^{\circ+7.839^{\circ}}, \quad \text { and } \quad \theta_{13}^{\nu} \simeq 7.27_{-2.824^{\circ}}^{\circ+2.012^{\circ}},
$$

but the $\mathrm{CP}$ violating phase $\delta$ remains unconstrained. As the recent $\mathrm{T} 2 \mathrm{~K}$ collaboration results [5] give more robust indication of a relatively large $\theta_{13}$ for $\delta_{\mathrm{CP}}=0$ :

$$
\begin{array}{cl}
0.03<\sin ^{2} 2 \theta_{13}<0.28, & \text { Normal Hierarchy (NH), } \\
0.04<\sin ^{2} 2 \theta_{13}<0.34, & \text { Inverted Hierarchy (IH), }
\end{array}
$$

the smallest mixing angle $\theta_{13}$ could be naturally produced

$$
4.987^{\circ}<\theta_{13}^{\nu}<15.974^{\circ}(\mathrm{NH}), \quad 5.769^{\circ}<\theta_{13}^{\nu}<17.834^{\circ}(\mathrm{IH}) .
$$


The best-fit values are $\sin ^{2} 2 \theta_{13}=0.11(\mathrm{NH})$ or $\sin ^{2} 2 \theta_{13}=0.14(\mathrm{IH})$, i.e.,

$$
\theta_{13}^{\nu}=9.685_{-6.289^{\circ}}^{\circ+4.698^{\circ}}(\mathrm{NH}) \quad \text { or } \theta_{13}^{\nu}=10.986_{-6.848^{\circ}}^{\circ+5.218^{\circ}}(\mathrm{IH}) .
$$

In recent years, lots of efforts have been devoted to explore a new theoretical framework to accommodate tiny neutrino masses and large flavor mixing angles. Among those studies which have tried to parametrize the PMNS matrix with only constant numbers, the proposal of bimaximal (BM) [30-35] and tribimaximal (TB) [36-40] mixing patterns are considered to be the most successful parametrizations and are mostly discussed. The specific forms are written as

$$
\begin{gathered}
U_{\mathrm{BM}}=\left(\begin{array}{ccc}
\sqrt{2} / 2 \sqrt{2} / 2 & 0 \\
-1 / 2 & 1 / 2 & \sqrt{2} / 2 \\
1 / 2 & -1 / 2 & \sqrt{2} / 2
\end{array}\right) P_{\nu}, \\
U_{\mathrm{TB}}=\left(\begin{array}{ccc}
2 / \sqrt{6} & 1 / \sqrt{3} & 0 \\
-1 / \sqrt{6} & 1 / \sqrt{3} & 1 / \sqrt{2} \\
1 / \sqrt{6} & -1 / \sqrt{3} & 1 / \sqrt{2}
\end{array}\right) P_{\nu},
\end{gathered}
$$

where $P_{\nu}=\operatorname{Diag}\left\{e^{-i \alpha / 2}, e^{-i \beta / 2}, 1\right\}$ includes two $\mathrm{CP}$-violating phases if the three neutrinos are Majorana fermions. These two scenarios give the prediction of neutrino mixing angles

$$
\begin{aligned}
& \theta_{23}^{\mathrm{BM}}=45^{\circ}, \quad \theta_{12}^{\mathrm{BM}}=45^{\circ}, \quad \theta_{13}^{\mathrm{BM}}=0^{\circ}, \\
& \theta_{23}^{\mathrm{TB}}=45^{\circ}, \quad \theta_{12}^{\mathrm{TB}}=35.26^{\circ}, \quad \theta_{13}^{\mathrm{TB}}=0^{\circ} \text {, }
\end{aligned}
$$

respectively in the standard parametrization of the $3 \times 3$ neutrino mixing matrix. In these two cases the Dirac CPviolating phase $\delta$ is absent, which leads to no $\mathrm{CP}$ violation in neutrino oscillations.

With the above experimental results as well as phenomenological deduction, combined with the quark-lepton complementarity (QLC) relations [8-19] given by

$$
\theta_{12}^{q}+\theta_{12}^{\nu}=45^{\circ}, \quad \theta_{23}^{q}+\theta_{23}^{\nu}=45^{\circ},
$$

we can directly compare the deviation of PMNS lepton mixing matrix from BM and TB mixing patterns in terms of three mixing angles:

$$
\left\{\begin{array}{lll}
\Delta_{23}^{\mathrm{BM}}=\left|\theta_{23}^{\nu}-\theta_{23}^{\mathrm{BM}}\right| \simeq 2.950^{\circ}, & & \sin \Delta_{23}^{\mathrm{BM}} \sim \mathcal{O}\left(\lambda^{2}\right), \\
\Delta_{12}^{\mathrm{BM}}=\left|\theta_{12}^{\nu}-\theta_{12}^{\mathrm{BM}}\right| \simeq 11.043^{\circ}, & & \sin \Delta_{12}^{\mathrm{BM}} \sim \mathcal{O}(\lambda), \\
\Delta_{13}^{\mathrm{BM}}=\left|\theta_{13}^{\nu}-\theta_{13}^{\mathrm{BM}}\right| \simeq 9.685^{\circ}\left(10.986^{\circ}\right), & \sin \Delta_{13}^{\mathrm{BM}} \sim \mathcal{O}(\lambda),
\end{array}\right.
$$

and

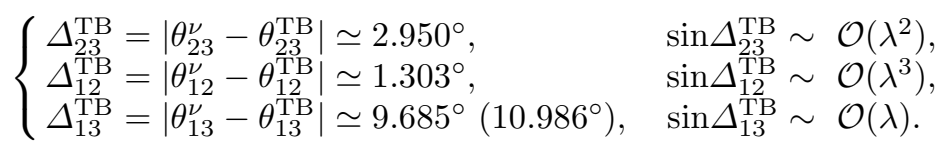

From above we find that the mixing angle $\Delta_{13}^{\nu} \sim \mathcal{O}(\lambda)$, which serves as a larger deviation from BM and TB mixing patterns according to the latest $\mathrm{T} 2 \mathrm{~K}$ result of larger $\theta_{13}$ for neutrino oscillation. This situation is different from our expectation $\Delta_{13}^{\nu} \sim \mathcal{O}\left(\lambda^{2}\right)$ or $\left(\lambda^{3}\right)$ with small $\theta_{13}$, which make it necessary to update the corresponding study [52] on expansions based on the deviation.

\subsection{Relating quarks and leptons}

In this section, we aim to explore the seemingly independent mixing patterns between quark and lepton sectors. The PMNS matrix depends generally on the charged lepton sector whose diagonalization leads to a charged lepton mixing matrix $U_{l}^{\dagger}$ which should be combined with the neutrino mixing matrix $U_{\nu}[43,44]$ :

$$
U_{\mathrm{PMNS}}=U_{l}^{\dagger} U_{\nu}
$$


Since the CKM matrix is quite near unit matrix, and the PMNS matrix has been considered to be close to bimaximal matrix and tribimaximal mixing matrix, it is interesting to assume that the deviation of the PMNS matrix from the BM and TB mixings can be described by the CKM matrix as generally discussed in reference [43,44], that is,

$$
U_{\mathrm{PMNS}} V_{\mathrm{CKM}}=U_{\mathrm{BM} / \mathrm{TB}}, \quad \text { or } \quad V_{\mathrm{CKM}} U_{\mathrm{PMNS}}=U_{\mathrm{BM} / \mathrm{TB}},
$$

which is equivalent to

$$
U_{\mathrm{PMNS}}=U_{\mathrm{BM} / \mathrm{TB}} V_{\mathrm{CKM}}^{\dagger}, \quad \text { or } \quad U_{\mathrm{PMNS}}=V_{\mathrm{CKM}}^{\dagger} U_{\mathrm{BM} / \mathrm{TB}} .
$$

Adopting similar interpretation, Ref. [45] provides explicit discussions of the following two cases for the relation between CKM and PMNS mixing matrices with $U_{\nu}$ the bimaximal mixing matrix:

$$
U_{\mathrm{PMNS}}=U_{\mathrm{BM}} V_{\mathrm{CKM}}^{\dagger}, \text { and } U_{\mathrm{PMNS}}=V_{\mathrm{CKM}}^{\dagger} U_{\mathrm{BM}}
$$

In the first case, substituting the corresponding matrices of $U_{\mathrm{BM}}$ and $V_{\mathrm{CKM}}$ one can calculate the moduli of each mixing element of $U_{\mathrm{PMNS}}$ and give

$$
\left|s_{13}^{\mathrm{PMNS}}\right|=\frac{\sqrt{2}}{2} A \lambda^{2} \sqrt{(\lambda-\lambda \rho-1)^{2}+(\lambda \eta)^{2}}=0.48 \lambda^{2} .
$$

Thus, we get the explicit value

$$
1.39^{\circ}<\theta_{13}<1.40^{\circ},
$$

assuming no $\mathrm{CP}$ violation. It is obvious that this region is excluded by the $\mathrm{T} 2 \mathrm{~K}$ results. Thus this scheme is not preferable judging by present experimental data.

In the second case, $U_{13}$ could be produced similarly

$$
\left|s_{13}^{\mathrm{PMNS}}\right|=\frac{\sqrt{2}}{2} \lambda \sqrt{\left[A \lambda^{2}(1-\rho)-1\right]^{2}+\left(A \lambda^{2} \eta\right)^{2}}=0.68 \lambda,
$$

and consequently

$$
8.79^{\circ}<\theta_{13}<8.84^{\circ} \text {. }
$$

Although the second case provides $\theta_{13}$ values more closer to the experimental data, it is proved in the original paper that this case does not accommodate the QLC relations, i.e., Eq. (13), very well [45].

We should point out that the multiplying relationship between PMNS and CKM matrices, such as Eq. (17), is not independent from different parametrizations of the charged lepton CKM matrix. The reason is that different choices of phase factors in $V_{\mathrm{CKM}}$ can produce differences in the predicted $U_{\text {PMNS. }}$ If we choose the leptonic mixing matrix $U_{\nu}$ to be tribimaximal pattern, for another choice of charged lepton mixing matrix $U_{l}^{\dagger}$ in the case of $U_{\mathrm{PMNS}}=U_{l}^{\dagger} U_{\mathrm{TB}}$, an Ansatz have been discussed [46,47] using a new parametrization [48]:

$$
U_{l}^{\dagger}=\left(\begin{array}{ccc}
1-\lambda^{2} / 2 & \lambda e^{i \delta} & h \lambda^{3} \\
-\lambda e^{-i \delta} & 1-\lambda^{2} / 2 & \left(f+h e^{-i \delta}\right) \lambda^{2} \\
f \lambda^{3} e^{-i \delta} & -\left(f+h e^{i \delta}\right) \lambda^{2} & 1
\end{array}\right)+\mathcal{O}\left(\lambda^{4}\right),
$$

where the parameters $A, \rho$ and $\eta$ in the Wolfenstein parametrization [25] are replaced by $f, h$ and $\delta$ in the new Wolfenstein-like one [48]. And the corresponding parameters read as:

$$
f=0.749_{-0.037}^{+0.034}, \quad h=0.309_{-0.012}^{+0.017}, \quad \lambda=0.22545 \pm 0.00065, \quad \delta=\left(89.6_{-0.86}^{+2.94}\right)^{\circ} .
$$

As we will see below, this matrix could be a candidate for the small deviation of the PMNS matrix from the TB mixing pattern.

By substituting the corresponding matrix in $U_{\mathrm{PMNS}}=U_{l}^{\dagger} U_{\nu}$, the leptonic mixing matrix corrected by the contributions from $U_{l}^{\dagger}$ can be written, up to order of $\lambda^{3}$, as $[46,47]$

$$
\begin{aligned}
U_{\mathrm{PMNS}} & =U_{\mathrm{TB}}+\left(\begin{array}{ccc}
-\frac{\lambda e^{i \delta}}{\sqrt{6}}-\frac{\lambda^{2}(1+h \lambda)}{\sqrt{6}} & \frac{\lambda e^{i \delta}}{\sqrt{3}}-\frac{\lambda^{2}(1-2 h \lambda)}{2 \sqrt{3}} & \frac{\lambda\left(h \lambda^{2}-e^{i \delta}\right)}{\sqrt{2}} \\
-\lambda \sqrt{\frac{2}{3}} e^{-i \delta}-\frac{\lambda^{2}\left(1-2 f-2 h e^{-i \delta}\right)}{2 \sqrt{6}} & -\frac{\lambda e^{-i \delta}}{\sqrt{3}}-\frac{\lambda^{2}\left(1-2 f-2 h \lambda e^{-i \delta}\right)}{2 \sqrt{3}} & \frac{\lambda^{2}\left(1+2 f+2 h e^{-i \delta}\right)}{2 \sqrt{2}} \\
\frac{\lambda^{2}\left(f+h e^{i \delta}+2 f \lambda e^{-i \delta}\right)}{\sqrt{6}} & -\frac{\lambda^{2}\left(f+h e^{i \delta}-f \lambda e^{-i \delta}\right)}{\sqrt{3}} & \frac{\lambda^{2}\left(f+h e^{i \delta}\right)}{\sqrt{2}}
\end{array}\right) P_{\nu} \\
& +\mathcal{O}\left(\lambda^{4}\right) .
\end{aligned}
$$


In this case, Ref. [46] adopts an alternative explicit form for tribimaximal mixing matrix with negative 21, 23 and 31 matrix elements, which is physically equivalent to Eq. (11). Thus, the smallest mixing angle $\theta_{13}$ can be obtained by $[46]$

$$
\sin \theta_{13}=\frac{\lambda}{\sqrt{2}}\left(1-h \lambda^{2} \cos \delta\right),
$$

with a non-vanishing $\theta_{13}=9.2^{\circ}$. Such a scheme, which has been proposed by Ahn-Cheng-Oh [46,47], provides a viable relation to connect quark and lepton mixing matrices in agreement with the new T2K results.

\section{Triminimal expansion}

After the above initiative study of T2K results, we found that the relatively large $\theta_{13}$ makes it necessary to update and expand the former works on triminimal expansion [51,52]. If the flavor mixing matrices are parametrized according to the hierarchical structure of mixing, it may reveal more physical information about the underlying theory. A good choice of this idea is the triminimal parametrization [49-52] with an approximation as the basis matrix to the lowest order. That is to express a mixing angle in the mixing matrix as the sum of a zeroth order angle $\theta^{0}$ and a small perturbation angle $\epsilon$ with

$$
\theta_{12}=\theta_{12}^{0}+\epsilon_{12}, \quad \theta_{23}=\theta_{23}^{0}+\epsilon_{23}, \quad \theta_{13}=\theta_{13}^{0}+\epsilon_{13} .
$$

With the deviations $\epsilon_{i j}$, one can expand the mixing matrix in powers of $\epsilon_{i j}$ while different choices of $\theta_{i j}^{0}$ lead to different basis.

According to new results of neutrino mixing angles, we can see that the deviation of PMNS mixing matrix from TB mixing pattern is more close to that of CKM mixing matrix from unit matrix if measured in terms of mixing angles. To be explicit, the comparison of deviation hierarchy is listed as follows for corresponding mixing angles: $\sin \Delta_{13}^{\mathrm{TB}} \sim \sin \Delta_{12}^{I} \sim \mathcal{O}(\lambda)$ and $\sin \Delta_{23}^{\mathrm{TB}} \sim \sin \Delta_{23}^{I} \sim \mathcal{O}\left(\lambda^{2}\right)$. From this aspect, the deviation hierarchy from BM and TB mixing patterns of different mixing angles can serve as the triminimal expansion basis in constructing the PMNS matrix.

\subsection{Expansion on bimaximal pattern}

To realize the triminimal expansion on BM mixing pattern, we assume that $\epsilon_{12}^{L}=-\lambda$ and $\epsilon_{23}^{L}=-A \lambda^{2}$ considering the suggestion of QLC [52], and $\epsilon_{13}^{L} e^{i \delta^{L}}=\lambda Z$ from the constraint of new T2K result as estimated in Sec. 2. To the third order in $\epsilon_{i j}^{L}$, the expansion is [52]

$$
\begin{aligned}
& U_{\mathrm{PMNS}}=U_{\mathrm{BM}}+\epsilon_{12}^{L}\left(\begin{array}{ccc}
-\frac{\sqrt{2}}{2} & \frac{\sqrt{2}}{2} & 0 \\
-\frac{1}{2} & -\frac{1}{2} & 0 \\
\frac{1}{2} & \frac{1}{2} & 0
\end{array}\right)+\epsilon_{23}^{L}\left(\begin{array}{ccc}
0 & 0 & 0 \\
\frac{1}{2} & -\frac{1}{2} & \frac{\sqrt{2}}{2} \\
\frac{1}{2} & -\frac{1}{2} & -\frac{\sqrt{2}}{2}
\end{array}\right)+\epsilon_{13}^{L}\left(\begin{array}{ccc}
0 & 0 & e^{-i \delta^{L}} \\
-\frac{1}{2} e^{i \delta^{L}} & -\frac{1}{2} e^{i \delta^{L}} & 0 \\
-\frac{1}{2} e^{i \delta^{L}} & -\frac{1}{2} e^{i \delta^{L}} & 0
\end{array}\right) \\
& +\left(\epsilon_{12}^{L}\right)^{2}\left(\begin{array}{ccc}
-\frac{\sqrt{2}}{4} & -\frac{\sqrt{2}}{4} & 0 \\
\frac{1}{4} & -\frac{1}{4} & 0 \\
-\frac{1}{4} & \frac{1}{4} & 0
\end{array}\right)+\left(\epsilon_{23}^{L}\right)^{2}\left(\begin{array}{ccc}
0 & 0 & 0 \\
\frac{1}{4} & -\frac{1}{4} & -\frac{\sqrt{2}}{4} \\
-\frac{1}{4} & \frac{1}{4} & -\frac{\sqrt{2}}{4}
\end{array}\right)+\left(\epsilon_{13}^{L}\right)^{2}\left(\begin{array}{ccc}
-\frac{\sqrt{2}}{4} & -\frac{\sqrt{2}}{4} & 0 \\
0 & 0 & -\frac{\sqrt{2}}{4} \\
0 & 0 & -\frac{\sqrt{2}}{4}
\end{array}\right) \\
& +\epsilon_{12}^{L} \epsilon_{23}^{L}\left(\begin{array}{ccc}
0 & 0 & 0 \\
\frac{1}{2} & \frac{1}{2} & 0 \\
\frac{1}{2} & \frac{1}{2} & 0
\end{array}\right)+\epsilon_{12}^{L} \epsilon_{13}^{L} e^{i \delta^{L}}\left(\begin{array}{ccc}
0 & 0 & 0 \\
\frac{1}{2} & -\frac{1}{2} & 0 \\
\frac{1}{2} & -\frac{1}{2} & 0
\end{array}\right)+\epsilon_{23}^{L} \epsilon_{13}^{L} e^{i \delta^{L}}\left(\begin{array}{ccc}
0 & 0 & 0 \\
-\frac{1}{2} & -\frac{1}{2} & 0 \\
\frac{1}{2} & \frac{1}{2} & 0
\end{array}\right) \\
& +\left(\epsilon_{12}^{L}\right)^{3}\left(\begin{array}{ccc}
\frac{\sqrt{2}}{12} & -\frac{\sqrt{2}}{12} & 0 \\
\frac{1}{12} & \frac{1}{12} & 0 \\
-\frac{1}{12} & -\frac{1}{12} & 0
\end{array}\right)+\left(\epsilon_{23}^{L}\right)^{3}\left(\begin{array}{ccc}
0 & 0 & 0 \\
-\frac{1}{12} & \frac{1}{12} & -\frac{\sqrt{2}}{12} \\
-\frac{1}{12} & \frac{1}{12} & \frac{\sqrt{2}}{12}
\end{array}\right)+\left(\epsilon_{13}^{L}\right)^{3}\left(\begin{array}{ccc}
0 & 0 & -\frac{1}{6} e^{-i \delta^{L}} \\
\frac{1}{12} e^{i \delta^{L}} & \frac{1}{12} e^{i \delta^{L}} & 0 \\
\frac{1}{12} e^{i \delta^{L}} & \frac{1}{12} e^{i \delta^{L}} & 0
\end{array}\right) \\
& +\epsilon_{12}^{L}\left(\epsilon_{23}^{L}\right)^{2}\left(\begin{array}{ccc}
0 & 0 & 0 \\
\frac{1}{4} & \frac{1}{4} & 0 \\
-\frac{1}{4} & -\frac{1}{4} & 0
\end{array}\right)+\left(\epsilon_{12}^{L}\right)^{2} \epsilon_{23}^{L}\left(\begin{array}{ccc}
0 & 0 & 0 \\
-\frac{1}{4} & \frac{1}{4} & 0 \\
-\frac{1}{4} & \frac{1}{4} & 0
\end{array}\right)+\epsilon_{12}^{L}\left(\epsilon_{13}^{L}\right)^{2}\left(\begin{array}{ccc}
\frac{\sqrt{2}}{4} & -\frac{\sqrt{2}}{4} & 0 \\
0 & 0 & 0 \\
0 & 0 & 0
\end{array}\right) \\
& +\left(\epsilon_{12}^{L}\right)^{2} \epsilon_{13}^{L} e^{i \delta^{L}}\left(\begin{array}{ccc}
0 & 0 & 0 \\
\frac{1}{4} & \frac{1}{4} & 0 \\
\frac{1}{4} & \frac{1}{4} & 0
\end{array}\right)+\epsilon_{23}^{L}\left(\epsilon_{13}^{L}\right)^{2}\left(\begin{array}{ccc}
0 & 0 & 0 \\
0 & 0 & -\frac{\sqrt{2}}{4} \\
0 & 0 & \frac{\sqrt{2}}{4}
\end{array}\right)+\left(\epsilon_{23}^{L}\right)^{2} \epsilon_{13}^{L} e^{i \delta^{L}}\left(\begin{array}{ccc}
0 & 0 & 0 \\
\frac{1}{4} & \frac{1}{4} & 0 \\
\frac{1}{4} & \frac{1}{4} & 0
\end{array}\right)
\end{aligned}
$$




$$
+\epsilon_{12}^{L} \epsilon_{23}^{L} \epsilon_{13}^{L} e^{i \delta^{L}}\left(\begin{array}{ccc}
0 & 0 & 0 \\
\frac{1}{2} & -\frac{1}{2} & 0 \\
-\frac{1}{2} & \frac{1}{2} & 0
\end{array}\right)+\mathcal{O}\left(\left(\epsilon_{i j}^{L}\right)^{4}\right) .
$$

Since the hierarchy is $\epsilon_{23}^{L} \sim\left(\epsilon_{12}^{L}\right)^{2}, \epsilon_{13} e^{i \delta^{L}} \sim \epsilon_{12}^{L}$, then to the third order in $\epsilon_{12}^{L}$, we have

$$
\begin{aligned}
& U_{\mathrm{PMNS}}=U_{\mathrm{BM}}+\epsilon_{12}^{L}\left(\begin{array}{ccc}
-\frac{\sqrt{2}}{2} & \frac{\sqrt{2}}{2} & 0 \\
-\frac{1}{2} & -\frac{1}{2} & 0 \\
\frac{1}{2} & \frac{1}{2} & 0
\end{array}\right)+\epsilon_{23}^{L}\left(\begin{array}{ccc}
0 & 0 & 0 \\
\frac{1}{2} & -\frac{1}{2} & \frac{\sqrt{2}}{2} \\
\frac{1}{2} & -\frac{1}{2} & -\frac{\sqrt{2}}{2}
\end{array}\right)+\epsilon_{13}^{L}\left(\begin{array}{ccc}
0 & 0 & e^{-i \delta^{L}} \\
-\frac{1}{2} e^{i \delta^{L}} & -\frac{1}{2} e^{i \delta^{L}} & 0 \\
-\frac{1}{2} e^{i \delta^{L}} & -\frac{1}{2} e^{i \delta^{L}} & 0
\end{array}\right) \\
& +\left(\epsilon_{12}^{L}\right)^{2}\left(\begin{array}{ccc}
-\frac{\sqrt{2}}{4} & -\frac{\sqrt{2}}{4} & 0 \\
\frac{1}{4} & -\frac{1}{4} & 0 \\
-\frac{1}{4} & \frac{1}{4} & 0
\end{array}\right)+\left(\epsilon_{13}^{L}\right)^{2}\left(\begin{array}{ccc}
-\frac{\sqrt{2}}{4} & -\frac{\sqrt{2}}{4} & 0 \\
0 & 0 & -\frac{\sqrt{2}}{4} \\
0 & 0 & -\frac{\sqrt{2}}{4}
\end{array}\right)+\epsilon_{12}^{L} \epsilon_{23}^{L}\left(\begin{array}{ccc}
0 & 0 & 0 \\
\frac{1}{2} & \frac{1}{2} & 0 \\
\frac{1}{2} & \frac{1}{2} & 0
\end{array}\right)+\epsilon_{12}^{L} \epsilon_{13}^{L} e^{i \delta^{L}}\left(\begin{array}{ccc}
0 & 0 & 0 \\
\frac{1}{2} & -\frac{1}{2} & 0 \\
\frac{1}{2} & -\frac{1}{2} & 0
\end{array}\right) \\
& +\epsilon_{23}^{L} \epsilon_{13}^{L} e^{i \delta^{L}}\left(\begin{array}{ccc}
0 & 0 & 0 \\
-\frac{1}{2} & -\frac{1}{2} & 0 \\
\frac{1}{2} & \frac{1}{2} & 0
\end{array}\right)+\left(\epsilon_{12}^{L}\right)^{3}\left(\begin{array}{ccc}
\frac{\sqrt{2}}{12} & -\frac{\sqrt{2}}{12} & 0 \\
\frac{1}{12} & \frac{1}{12} & 0 \\
-\frac{1}{12} & -\frac{1}{12} & 0
\end{array}\right)+\left(\epsilon_{13}^{L}\right)^{3}\left(\begin{array}{ccc}
0 & 0 & -\frac{1}{6} e^{-i \delta^{L}} \\
\frac{1}{12} e^{i \delta^{L}} & \frac{1}{12} e^{i \delta^{L}} & 0 \\
\frac{1}{12} e^{i \delta^{L}} & \frac{1}{12} e^{i \delta^{L}} & 0
\end{array}\right) \\
& +\epsilon_{12}^{L}\left(\epsilon_{13}^{L}\right)^{2}\left(\begin{array}{ccc}
\frac{\sqrt{2}}{4} & -\frac{\sqrt{2}}{4} & 0 \\
0 & 0 & 0 \\
0 & 0 & 0
\end{array}\right)+\left(\epsilon_{12}^{L}\right)^{2} \epsilon_{13}^{L} e^{i \delta^{L}}\left(\begin{array}{ccc}
0 & 0 & 0 \\
\frac{1}{4} & \frac{1}{4} & 0 \\
\frac{1}{4} & \frac{1}{4} & 0
\end{array}\right)+\mathcal{O}\left(\left(\epsilon_{i j}^{L}\right)^{4}\right) \\
& =U_{\mathrm{BM}}-\lambda\left(\begin{array}{ccc}
-\frac{\sqrt{2}}{2} & \frac{\sqrt{2}}{2} & -Z e^{-i 2 \delta^{L}} \\
-\frac{1}{2}(1-Z) & -\frac{1}{2}(1-Z) & 0 \\
\frac{1}{2}(1+Z) & \frac{1}{2}(1+Z) & 0
\end{array}\right) \\
& +\lambda^{2}\left(\begin{array}{ccc}
-\frac{\sqrt{2}}{4}\left(1+Z^{2} e^{-i 2 \delta^{L}}\right) & -\frac{\sqrt{2}}{4}\left(1+Z^{2} e^{-i 2 \delta^{L}}\right) & 0 \\
-\frac{1}{2}\left(A+Z-\frac{1}{2}\right) & \frac{1}{2}\left(A+Z-\frac{1}{2}\right) & -\frac{\sqrt{2}}{\sqrt{2}}\left(A+\frac{1}{2} Z^{2} e^{-i 2 \delta^{L}}\right) \\
-\frac{1}{2}\left(A+Z+\frac{1}{2}\right) & \frac{1}{2}\left(A+Z+\frac{1}{2}\right) & \frac{\sqrt{2}}{2}\left(A-\frac{1}{2} Z^{2} e^{-i 2 \delta^{L}}\right)
\end{array}\right) \\
& +\lambda^{3}\left(\begin{array}{ccc}
-\frac{\sqrt{2}}{4}\left(\frac{1}{3}+Z^{2} e^{-i 2 \delta^{L}}\right) & \frac{\sqrt{2}}{4}\left(\frac{1}{3}+Z^{2} e^{-i 2 \delta^{L}}\right) & -\frac{1}{6} e^{-i 4 \delta^{L}} \\
\frac{1}{12}\left(6 A Z+6 A+3 Z+Z^{3} e^{-i 2 \delta^{L}}-1\right) & \frac{1}{12}\left(6 A Z+6 A+3 Z+Z^{3} e^{-i 2 \delta^{L}}-1\right) & 0 \\
\frac{1}{12}\left(-6 A Z+6 A+3 Z+Z^{3} e^{-i 2 \delta^{L}}+1\right) \frac{1}{12}\left(-6 A Z+6 A+3 Z+Z^{3} e^{-i 2 \delta^{L}}+1\right) & 0
\end{array}\right) \\
& +\mathcal{O}\left(\lambda^{4}\right) \text {. }
\end{aligned}
$$

\subsection{Expansion on tribimaximal pattern}

If we start expansion with an alternative TB mixing form, the triminimal parametrization of the PMNS matrix is obtained as $[49,50]$ to the second order in $\epsilon_{i j}^{L}$ :

$$
\begin{aligned}
U_{\mathrm{PMNS}} & =U_{\mathrm{TB}}+\epsilon_{12}^{L}\left(\begin{array}{ccc}
-\frac{1}{\sqrt{3}} & \frac{2}{\sqrt{6}} & 0 \\
-\frac{1}{\sqrt{3}} & -\frac{1}{\sqrt{6}} & 0 \\
\frac{1}{\sqrt{3}} & \frac{1}{\sqrt{6}} & 0
\end{array}\right)+\epsilon_{23}^{L}\left(\begin{array}{ccc}
0 & 0 & 0 \\
\frac{1}{\sqrt{6}}-\frac{1}{\sqrt{3}} & \frac{1}{\sqrt{2}} \\
\frac{1}{\sqrt{6}}-\frac{1}{\sqrt{3}}-\frac{1}{\sqrt{2}}
\end{array}\right)+\epsilon_{13}^{L}\left(\begin{array}{ccc}
0 & 0 & e^{-i \delta^{L}} \\
-\frac{1}{\sqrt{3}} e^{i \delta^{L}} & -\frac{1}{\sqrt{6}} e^{i \delta^{L}} & 0 \\
-\frac{1}{\sqrt{3}} e^{i \delta^{L}} & -\frac{1}{\sqrt{6}} e^{i \delta^{L}} & 0
\end{array}\right) \\
& +\left(\epsilon_{12}^{L}\right)^{2}\left(\begin{array}{ccc}
-\frac{1}{\sqrt{6}} & -\frac{1}{2 \sqrt{3}} & 0 \\
\frac{1}{2 \sqrt{6}} & -\frac{1}{2 \sqrt{3}} & 0 \\
-\frac{1}{2 \sqrt{6}} & \frac{1}{2 \sqrt{3}} & 0
\end{array}\right)+\left(\epsilon_{23}^{L}\right)^{2}\left(\begin{array}{ccc}
0 & 0 & 0 \\
\frac{1}{2 \sqrt{6}} & -\frac{1}{2 \sqrt{3}}-\frac{1}{2 \sqrt{2}} \\
-\frac{1}{2 \sqrt{6}} & \frac{1}{2 \sqrt{3}}-\frac{1}{2 \sqrt{2}}
\end{array}\right)+\left(\epsilon_{13}^{L}\right)^{2}\left(\begin{array}{ccc}
-\frac{1}{\sqrt{6}}-\frac{1}{2 \sqrt{3}} & 0 \\
0 & 0 & -\frac{1}{2 \sqrt{2}} \\
0 & 0 & -\frac{1}{2 \sqrt{2}}
\end{array}\right) \\
& +\epsilon_{12}^{L} \epsilon_{23}^{L}\left(\begin{array}{ccc}
0 & 0 \\
\frac{1}{\sqrt{3}} \frac{1}{\sqrt{6}} & 0 \\
\frac{1}{\sqrt{3}} \frac{1}{\sqrt{6}} & 0
\end{array}\right)+\epsilon_{12}^{L} \epsilon_{13}^{L} e^{i \delta^{L}}\left(\begin{array}{ccc}
0 & 0 \\
\frac{1}{\sqrt{6}}-\frac{1}{\sqrt{3}} & 0 \\
\frac{1}{\sqrt{6}}-\frac{1}{\sqrt{3}} & 0
\end{array}\right)+\epsilon_{23}^{L} \epsilon_{13}^{L} e^{i \delta^{L}}\left(\begin{array}{ccc}
0 & 0 \\
-\frac{1}{\sqrt{3}}-\frac{1}{\sqrt{6}} & 0 \\
\frac{1}{\sqrt{3}} & \frac{1}{\sqrt{6}} & 0
\end{array}\right)+\mathcal{O}\left(\left(\epsilon_{i j}^{L}\right)^{3}\right),
\end{aligned}
$$

where $\epsilon_{23}^{L}, \epsilon_{13}^{L}$, and $\delta^{L}$ are the same parameters as parametrization of the PMNS matrix just like in the BM triminimal expansion case, whereas $\epsilon_{12}^{L}$ is not. This set of expansion parameters is certainly better than the one in the previous section if convergency is the criteria of the expansion. With the replacements $\epsilon_{12}^{L}=B \lambda^{3}, \epsilon_{23}^{L}=-A \lambda^{2}$ and $\epsilon_{13}^{L} e^{i \delta^{L}}=\lambda Z$, the hierarchy is $\epsilon_{12}^{L} \sim\left(\epsilon_{13}^{L}\right)^{3}$ and $\epsilon_{23}^{L} \sim\left(\epsilon_{13}^{L}\right)^{2}$. The parameters $A$ and $\lambda$ are the same Wolfenstein parameters as those 
in the BM case, argued from the aspect of QLC relations, and $B$ is a new parameter, which is of order $\mathcal{O}(1)$, adjusted to fit the data according to the estimate in Sec. II. Thus to the third order of $\epsilon_{13}^{L}$ :

$$
\begin{aligned}
U_{\mathrm{PMNS}} & =U_{\mathrm{TB}}+\epsilon_{12}^{L}\left(\begin{array}{ccc}
-\frac{1}{\sqrt{3}} & \frac{2}{\sqrt{6}} & 0 \\
-\frac{1}{\sqrt{3}} & -\frac{1}{\sqrt{6}} & 0 \\
\frac{1}{\sqrt{3}} & \frac{1}{\sqrt{6}} & 0
\end{array}\right)+\epsilon_{23}^{L}\left(\begin{array}{ccc}
0 & 0 & 0 \\
\frac{1}{\sqrt{6}}-\frac{1}{\sqrt{3}} & \frac{1}{\sqrt{2}} \\
\frac{1}{\sqrt{6}}-\frac{1}{\sqrt{3}}-\frac{1}{\sqrt{2}}
\end{array}\right)+\epsilon_{13}^{L}\left(\begin{array}{ccc}
0 & 0 & e^{-i \delta^{L}} \\
-\frac{1}{\sqrt{3}} e^{i \delta^{L}} & -\frac{1}{\sqrt{6}} e^{i \delta^{L}} & 0 \\
-\frac{1}{\sqrt{3}} e^{i \delta^{L}}-\frac{1}{\sqrt{6}} e^{i \delta^{L}} & 0
\end{array}\right) \\
& +\left(\epsilon_{13}^{L}\right)^{2}\left(\begin{array}{ccc}
-\frac{1}{\sqrt{6}}-\frac{1}{2 \sqrt{3}} & 0 \\
0 & 0 & -\frac{1}{2 \sqrt{2}} \\
0 & 0 & -\frac{1}{2 \sqrt{2}}
\end{array}\right)+\epsilon_{23}^{L} \epsilon_{13}^{L} e^{i \delta^{L}}\left(\begin{array}{ccc}
0 & 0 & 0 \\
-\frac{1}{\sqrt{3}}-\frac{1}{\sqrt{6}} & 0 \\
\frac{1}{\sqrt{3}} & \frac{1}{\sqrt{6}} & 0
\end{array}\right)+\mathcal{O}\left(\left(\epsilon_{13}^{L}\right)^{4}\right) \\
& =U_{\mathrm{TB}}+\lambda\left(\begin{array}{ccc}
0 & 0 & e^{-i 2 \delta^{L}} \\
-\frac{1}{\sqrt{3}} Z-\frac{1}{\sqrt{6}} Z & 0 \\
-\frac{1}{\sqrt{3}} Z-\frac{1}{\sqrt{6}} Z & 0
\end{array}\right)-\lambda^{2}\left(\begin{array}{ccc}
\frac{1}{\sqrt{6}} Z^{2} e^{-i 2 \delta^{L}} & \frac{1}{2 \sqrt{3}} Z^{2} e^{-i 2 \delta^{L}} & 0 \\
\frac{1}{\sqrt{6}} A & -\frac{1}{\sqrt{3}} A & \frac{1}{2 \sqrt{2}}\left(2 A+Z^{2} e^{-i 2 \delta^{L}}\right) \\
\frac{1}{\sqrt{6}} A & -\frac{1}{\sqrt{3}} A & -\frac{1}{2 \sqrt{2}}\left(2 A-Z^{2} e^{-i 2 \delta^{L}}\right)
\end{array}\right) \\
& +\lambda^{3}\left(\begin{array}{ccc}
-\frac{1}{\sqrt{3}} B & \frac{2}{\sqrt{3}} B & 0 \\
-\frac{1}{\sqrt{3}}(B-A Z)-\frac{1}{\sqrt{6}}(B-A Z) & \frac{1}{\sqrt{6}}(B-A Z) & 0
\end{array}\right)+\mathcal{O}\left(\lambda^{4}\right) .
\end{aligned}
$$

It is novel that the deviations of the lepton sector are explicitly illustrated in orders of Wolfenstein parameter $\lambda$ from the quark sector, along with the idea to understand both the quark and lepton mixing patterns in a unified manner [51,52].

\section{A phenomenological proposal of new mixing pattern}

From previous global fits of neutrino mixing angles,

$$
\theta_{12}^{\nu} \simeq 33.957_{-2.143^{\circ}}^{\circ+2.434^{\circ}}, \quad \theta_{23}^{\nu} \simeq 43.050_{-5.834^{\circ}}^{\circ},
$$

together with the latest T2K implication:

$$
\theta_{13}^{\nu}=9.685_{-6.289^{\circ}}^{\circ+4.698^{\circ}}(\mathrm{NH}) \quad \text { or } \theta_{13}^{\nu}=10.986_{-6.848^{\circ}}^{\circ+5.218^{\circ}}(\mathrm{IH}),
$$

we can find a self-complementary relation between the mixing angles

$$
\theta_{12}^{\nu}+\theta_{13}^{\nu} \simeq \theta_{23}^{\nu} \simeq 45^{\circ} .
$$

This leads to a proposal for a new mixing pattern which is closer to the experimental data than BM and TM patterns. To construct such a new mixing pattern, we begin with the assumption that

$$
\begin{gathered}
\sin ^{2} \theta_{23}=\frac{1}{2}, \\
\sin ^{2} \theta_{12}=\frac{1}{3}, \\
\sin ^{2} \theta_{13}=\sin ^{2}\left(\theta_{23}-\theta_{12}\right)=\frac{1}{2}-\frac{\sqrt{2}}{3} .
\end{gathered}
$$

Thus the new mixing matrix could be given as

$$
\begin{aligned}
U_{\mathrm{NM}} & =\left(\begin{array}{ccc}
\frac{\sqrt{2}}{\sqrt{3}} \sqrt{\frac{1}{2}+\frac{\sqrt{2}}{3}} & \frac{1}{\sqrt{3}} \sqrt{\frac{1}{2}+\frac{\sqrt{2}}{3}} & \frac{1}{\sqrt{3}}-\frac{1}{\sqrt{6}} \\
-\frac{1}{3}+\frac{\sqrt{2}}{6}-\frac{1}{\sqrt{6}} & -\frac{\sqrt{2}}{6}+\frac{1}{6}+\frac{1}{\sqrt{3}} \frac{\sqrt{2}}{2} \sqrt{\frac{1}{2}+\frac{\sqrt{2}}{3}} \\
-\frac{1}{3}+\frac{\sqrt{2}}{6}+\frac{1}{\sqrt{6}} & -\frac{\sqrt{2}}{6}+\frac{1}{6}-\frac{1}{\sqrt{3}} \frac{\sqrt{2}}{2} \sqrt{\frac{1}{2}+\frac{\sqrt{2}}{3}}
\end{array}\right) \\
& \simeq\left(\begin{array}{ccc}
0.8047 & 0.5690 & 0.1691 \\
-0.5059 & 0.5083 & 0.6969 \\
0.3106 & -0.6464 & 0.6969
\end{array}\right) .
\end{aligned}
$$

One can easily see that for this new mixing pattern, the moduli of the mixing matrix elements are compatible with the results from global fits [27-29]. Generally, the mixing matrix is expanded as below. With the replacements 
$\epsilon_{12}^{L}=B \lambda^{3}, \epsilon_{23}^{L}=-A \lambda^{2}$, and $\epsilon_{13}^{L} e^{i \delta_{L}}=\lambda^{3} Z^{\prime}$ or $\lambda^{2} Z^{\prime}$ defined according to the hierarchical structure, the expansion could be obtained to the order of $\mathcal{O}\left(\lambda^{3}\right)$ :

$$
\begin{aligned}
& U_{\mathrm{PMNS}}=U_{\mathrm{NM}}+\epsilon_{12}\left(\begin{array}{ccc}
-s_{12}^{0} c_{13}^{0} & c_{12}^{0} c_{13}^{0} & 0 \\
s_{12}^{0} s_{13}^{0} s_{22}^{0} e^{i \delta}-c_{12}^{0} c_{23}^{0}-c_{12}^{0} s_{13}^{0} s_{23}^{0} e^{i \delta}-s_{12}^{0} c_{23}^{0} & 0 \\
s_{12}^{0} s_{13}^{0} c_{23}^{0} e^{i \delta}+c_{12}^{0} s_{23}^{0}-c_{12}^{0} s_{13}^{0} c_{23}^{0} e^{i \delta}+s_{12}^{0} s_{23}^{0} & 0
\end{array}\right) \\
& +\epsilon_{23}\left(\begin{array}{ccc}
0 & 0 & 0 \\
-s_{13}^{0} c_{12}^{0} c_{23}^{0} e^{i \delta}+s_{23}^{0} s_{12}^{0}-s_{12}^{0} s_{13}^{0} c_{23}^{0} e^{i \delta}-s_{23}^{0} c_{12}^{0} & c_{13}^{0} c_{23}^{0} \\
c_{12}^{0} s_{23}^{0} s_{13}^{0} e^{i \delta}+c_{23}^{0} s_{12}^{0} & s_{12}^{0} s_{23}^{0} s_{13}^{0} e^{i \delta}-c_{12}^{0} c_{23}^{0} & c_{12}^{0} s_{23}^{0}
\end{array}\right) \\
& +\epsilon_{13}\left(\begin{array}{ccc}
-c_{12}^{0} s_{13}^{0} & -s_{12}^{0} s_{13}^{0} & c_{13}^{0} e^{-i \delta} \\
-c_{12}^{0} c_{13}^{0} s_{23}^{0} e^{i \delta} & -c_{13}^{0} s_{23}^{0} s_{12}^{0} e^{i \delta} & -s_{13}^{0} s_{23}^{0} \\
-c_{12}^{0} c_{13}^{0} c_{23}^{0} e^{i \delta} & -s_{12}^{0} c_{13}^{0} c_{23}^{0} e^{i \delta}-s_{13}^{0} c_{23}^{0}
\end{array}\right)+\mathcal{O}\left(\lambda^{4}\right),
\end{aligned}
$$

where $s_{i j}^{0}=\sin \theta_{i j}, c_{i j}^{0}=\cos \theta_{i j}$ with $\theta_{i j}$ denotes the complementary mixing angles proposed in Eq. (35). We see that this expansion based on the new mixing pattern is much simpler compared with the triminimal expansions based on either BM or TM mixing patterns in the above section. The leading order basis matrix $U_{\mathrm{NM}}$ is much closer to the experimental fits.

\section{Summary}

In summary, we re-analyzed the neutrino mixing patterns according to the latest T2K result for a larger mixing angle $\theta_{13}$, and found that the deviation of the neutrino mixing pattern from bimaximal (BM) and tribimaximal (TB) patterns become larger than previously expected. We also examined relations connecting quark and lepton mixing matrices and it turned out that several schemes can still accommodate the latest T2K result nicely. As a necessary update of previous works, we made new triminimal expansions of the PMNS mixing matrix based on BM and TB mixing patterns in terms of a more reasonable $\lambda$ hierarchy. From the phenomenological point of view, we also proposed a new mixing pattern with a self-complementary relation between the mixing angles $\theta_{12}^{\nu}+\theta_{13}^{\nu} \simeq 45^{\circ}$, and concluded that such a mixing pattern works well in providing rather good descriptions to the data at least with a precision of $\mathcal{O}\left(\lambda^{2}\right)$. Thus the new T2K result can enrich our understanding of neutrino properties.

This work is partially supported by National Natural Science Foundation of China (Grants No. 11021092, No. 10975003, No. 11035003, and No. 11120101004) and by Peking University Visiting Scholar Program for Graduate Students.

\section{References}

1. SNO Collaboration, Q.R. Ahmad et al., Phys. Rev. Lett. 89, 011301 (2002).

2. For a review, see: C.K. Jung et al., Ann. Rev. Nucl. Part. Sci. 51, 451 (2001).

3. KamLAND Collaboration, K. Eguchi et al., Phys. Rev. Lett. 90, 021802 (2003).

4. K2K Collaboration, M.H. Ahn et al., Phys. Rev. Lett. 90, 041801 (2003).

5. The T2K Collaboration, K. Abe et al., Phys. Rev. Lett. 107, 041801 (2011)

6. Z. z. Xing, arXiv:1106.3244 [hep-ph].

7. N. Qin and B.-Q. Ma, Phys. Lett. B 702, 143 (2011).

8. A. Y. Smirnov, arXiv:hep-ph/0402264.

9. M. Raidal, Phys. Rev. Lett. 93, 161801 (2004).

10. H. Minakata, arXiv:hep-ph/0505262.

11. P. H. Frampton and R. N. Mohapatra, JHEP 0501, 025 (2005).

12. N. Li and B.-Q. Ma, Phys. Rev. D 71, 097301 (2005).

13. R. N. Mohapatra, Phys. Lett. B 618, 150 (2005).

14. J. Ferrandis and S. Pakvasa, Phys. Rev. D71, 033004 (2005).

15. S.K. Kang, C.S. Kim and J. Lee, Phys. Lett. B619, 129 (2005).

16. S. Antusch, S.F. King and R.N. Mohapatra, Phys. Lett. B618, 150 (2005).

17. M.A. Schmidt and A.Y. Smirnov, Phys. Rev. D74, 113003 (2006).

18. K. A. Hochmuth and W. Rodejohann, Phys. Rev. D 75, 073001 (2007)

19. F. Plentinger, G. Seidl and W. Winter, Phys. Rev. D76, 113003 (2007).

20. N. Cabibbo, Phys. Rev. Lett. 10, 531 (1963);

21. M. Kobayashi and T. Maskawa, Prog. Theor. Phys. 49, 652 (1973). 
22. Z. Maki, M. Nakagawa and S. Sakata, Prog. Theor. Phys. 28, 870 (1962).

23. B. Pontecorvo, Sov. Phys. JETP 26, 984 (1968) [Zh. Eksp. Teor. Fiz. 53, 1717 (1967)].

24. L.L. Chau and W.Y. Keung, Phys. Rev. Lett. 53, 1802 (1984).

25. L. Wolfenstein, Phys. Rev. Lett. 51, 1945 (1983).

26. K. Nakamura et al. (Particle Data Group), J. Phys. G 37, 075021 (2010).

27. Y. j. Zheng, Phys. Rev. D 81, 073009 (2010)

28. See, e.g., G. L. Fogli, E. Lisi, A. Marrone, A. Palazzo and A. M. Rotunno, Nucl. Phys. Proc. Suppl. 188, 27 (2009).

29. T. Schwetz, M. A. Tortola and J. W. F. Valle, New J. Phys. 10, 113011 (2008).

30. F. Vissani, hep-ph/9708483.

31. V.D. Barger, S.Pakvasa, T.J. Weiler, and K. Whisnant, Phys. Lett. B437, 107 (1998).

32. A.J. Baltz, A.S. Goldhaber, and M. Goldhaber, Phys. Rev. Lett. 81, 5730 (1998).

33. I. Stancu and D.V. Ahluwalia, Phys. Lett. B460, 431 (1999).

34. H. Georgi and S.L. Glashow, Phy. Rev. D61, 097301 (2000).

35. N. Li and B.-Q. Ma, Phys. Lett. B 600, 248 (2004)

36. P.F. Harrison, D.H. Perkins, and W.G. Scott, Phys. Lett. B458, 79 (1999); Phys. Lett. B530, 167 (2002);

37. Z.Z. Xing, Phys. Lett. B533, 85 (2002);

38. P.F. Harrison and W.G. Scott, Phys. Lett. B535, 163 (2002); Phys. Lett. B557, 76 (2003);

39. X.-G. He and A. Zee, Phys. Lett. B560, 87 (2003);

40. N. Li and B.-Q. Ma, Phys. Rev. D 71, 017302 (2005)

41. L. Wolfenstein, Phys. Rev. D18, 958 (1978);

42. Y. Yamanaka, H. Sugawara, and S. Pakvasa, Phys. Rev. D25, 1895 (1982); D29, 2135(E) (1984).

43. H. Minakata and A. Y. Smirnov, Phys. Rev. D 70, 073009 (2004)

44. K. A. Hochmuth, S. T. Petcov and W. Rodejohann, Phys. Lett. B 654, 177 (2007)

45. N. Li and B.-Q. Ma, Eur. Phys. J. C 42, 17 (2005)

46. Y. H. Ahn, H. Y. Cheng and S. Oh, arXiv:1105.4460 [hep-ph].

47. S. Goswami, S. T. Petcov, S. Ray and W. Rodejohann, Phys. Rev. D 80, 053013 (2009)

48. N. Qin and B.-Q. Ma, Phys. Lett. B 695, 194 (2011), Phys. Rev. D 83, 033006 (2011)

49. S. Pakvasa, W. Rodejohann and T.J. Weiler, Phy. Rev. Lett. 100, 111801 (2008).

50. S.F. King, Phys. Lett. B659, 244 (2008).

51. X.-G. He, S.-W. Li and B.-Q. Ma, Phys. Rev. D 78, 111301(R) (2008).

52. X.-G. He, S.-W. Li and B.-Q. Ma, Phys. Rev. D 79, 073001 (2009). 